\title{
Self-Enforcement Strategy for Energy-Efficient Relaying in Wireless Ad-hoc Networks
}

\author{
Michał Kucharzak Jacek Kibiłda Marcin Filo Radosław Piesiewicz \\ ICT Research Center \\ Wrocławskie Centrum Badań EIT+, Wrocław, Poland \\ \{michal.kucharzak, jacek.kibilda, marcin.filo, radoslaw.piesiewicz\}@eitplus.pl
}

\begin{abstract}
In this paper we propose and examine 802.11b-based MAC protocol for wireless ad-hoc relay networks. The objective of the proposed protocol is to incorporate self-enforcing strategy to achieve energy efficiency in function of joules required for providing data transmission between end users. Regarding energy requirements for transmitting and receiving frames, it is desirable for every node in a wireless system, to get access to the channel as fast as possible and occupy it for a reasonably short period. The protocol design addresses the relay's self-selection mechanism which is based on internal motivation. The motivation refers to willingness of candidate relay to aid the ongoing transmission with regard to possibility of being awarded faster access to wireless medium, yielding decrease in energy consumption spent in waiting mode. The paper reports major improvements in terms of energy efficiency for most of the presented scenarios, while using relaying protocol in contrast to non-relaying $802.11 \mathrm{~b}$.
\end{abstract}

Index Terms-relay networks, energy efficiency, green radio.

\section{INTRODUCTION}

Relaying transfers the concept of spatial diversity to much more decentralized and distributed wireless communication systems by creating virtual antenna arrays [7], [14], [20]. Such techniques incorporate additional relays in between two wireless nodes interested in data transmission. In principle, relaying provides robustness against fading, packet collision and mobility, which yields higher throughput or decreased delay in the system. Wide range of successful protocols designed for relaying is implemented at physical and MAC layer. They comprise simple and cooperative strategies such as cooperative beamforming [17], [23], distributed space time coding [12], [15], [21], [22], or selective schemes [1], [2], [5], [6], [10], [24], where single or multiple relays are selected to collaborate on information transmission.

Abstracting from technical implementations of wireless protocols, every transmission requires energy consumption. Considering relayed IEEE 802.11b-based ad-hoc systems, energy spent for realization of data transmission might include extra expenses as a consequence of using additional relays in between the source and destination. However, for $802.11 \mathrm{~b}$ standard, it might be assumed, that the radio's transmit mode has marginal impact on overall energy consumption, while other modes (receive and idle) are responsible for the most of consumed energy [3]. This fact is a driver for the idea of relaying enforced by self motivation in order to increase energy efficiency.

In this paper we apply self-enforcing strategy combined with an opportunistic relaying and propose a simple relaying MAC protocol with fixed contention window. In order to compare energy effectiveness of this method, we employ IEEE $802.11 \mathrm{~b}$ protocol augmented with capability of simple relaying triggered by users' self motivation. Simulation results prove that self-enforced relaying in ad-hoc networks provides explicit savings in terms of consumed energy.

\section{Self-Enforcement Strategy}

Basically, energy efficiency refers to savings in energy consumption in the network. However, being more precise, the "efficiency" can be defined in several separate ways regarding various requirements for the system. On the one hand, many wireless systems are designed to realize common goals (e.g. wireless sensor networks) but on the other hand, there are some systems like 802.11, which comprise separate transmissions and set of users which aim at realizing their own demands. These two examples are introduced into discussions in order to explain different node's attitudes which influence the system performance and feasibility of various methods. Systems with globally common goal usually implement mechanisms that force specific behaviour to achieve the desired result. Contrary, the assumption of common goal for system of users with individual demands and shared wireless medium is too idealized. The most appropriate way to "enforce" determined user behaviour is implementation of "self-enforcing" strategy. In self-enforcement scheme, users are internally motivated to perform particular behaviour, e.g. they contribute to network energy saving while it is beneficial for them. The mechanism is the most efficient if user's motivation is derived from its local selfish goals.

\section{A. Self-Enforcing Relaying}

Assuming capability of multi-rate in wireless communication systems, if channel quality is sufficiently high, shorter transmission duration time is achieved by employing higher modulation schemes. However, as noted in [11], multi-rate advantage might as well cause fairness problem. It is obvious that the low data rate stations will use more channel time than the high data rate stations for the same volume of data. This affects the system in two general ways: stations with low data rate experience poor service and by increased channel occupation time, they also reduce the bandwidth of high data rate stations, decreasing the effective throughput of 
the network. Both reduced throughput and increased channel occupation time, cause the growth of energy consumption in the network.

Let us consider the following network example:

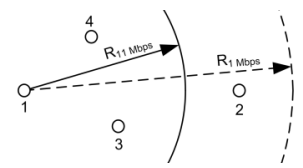

Figure 1. Simple network scenario.

In the scenario in Fig. 1, two separate transmissions take place between nodes 1-2 and 3-4. Having the shared wireless medium for both transmissions, nodes are supposed to compete for access to the channel. It is intuitive that, while nodes 12 are sender and receiver, nodes 3-4 stay idle, buffering the packets until the channel becomes available. This waiting time, basically depends on transmission duration time between the pair 1-2. If the data rate on link 1-2 is low (e.g. $1 \mathrm{Mbps}$ ), waiting time of remaining nodes increases (by $1 / R_{1 M b p s}$ times length of the frame plus total control-based procedure), thus causing the nodes to consume their energy for remaining idle. If the nodes could spend some additional energy in transmission mode and help to relay data, some overall energy savings might be provided by decreasing total transmission time and decreasing time for waiting before sending own data. Transmission time is decreased if $\left(1 / R_{s r}+1 / R_{r d}\right)<1 / R_{s d}$, where $R_{s d}, R_{s r}$ and $R_{r d}$ refer to data rates between source and destination, source and relay and relay and destination, respectively. If the data rate condition is fulfilled, nodes 3 and 4 are self-motivated to help in order to gain faster access to the channel. The overall energy efficiency might be improved if the total energy consumption for transmission via relay is less than energy consumed for direct transmission and energy spent on waiting for free media.

\section{Protocol Design}

The proposed energy-efficient self-enforcing relaying protocol is based on the ad-hoc mode in the Distributed Coordination Function (DCF) of 802.11b. For simplicity, control frames are transmitted at the lowest rate and data frames are adaptively broadcasted with the highest possible data rate. Transmitting stations choose the best modulation scheme based on the received signal-to-noise ratio (SNR). Transmission powers are fixed and defined in a static way.

With the protocol every node which is interested in data transmission waits for free channel. It simultaneously and promiscuously listens to all ongoing RTS and CTS packets. By measuring signal quality of RTS and CTS packets, node can calculate maximum data rate on both links between itself and senders of RTS and CTS. It is assumed that the forward and backward channels between nodes are symmetrical due to the reciprocity theorem [19]. If node has to wait certain amount of time for access to the medium because of other ongoing transmission, it might reduce transmission time between other nodes by employing self-enforcement relaying.
The frames exchange sequence of self-enforcing relaying protocol is illustrated in Fig. 2.

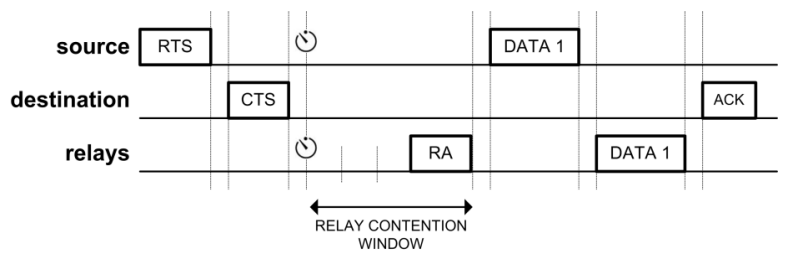

Figure 2. Self-enforcing relaying protocol frame exchange sequence.

Transaction starts with RTS-CTS handshaking as standarized in [13]. All nodes interested in channel access measure RTS and CTS signal qualities and calculate maximum data rate which can be applied on links between the source the destination. This concept of channel estimation by measurement of control packets signal is common for most relaying MAC protocols. Next, source node and all potential relays start their internal timers. Since then, source and relays compete in relay selection phase and their timers are chosen randomly among values from contention sub-window. The timer duration expires faster if better end-to-end data rate might be provided. In case that, transmission duration time cannot be increased by employing relaying (direct link quality provides data rates either $11 \mathrm{Mbps}$ or $5.5 \mathrm{Mbps}$ ), the source starts sending DATA frame immediately after SIFS. Idea of contention window appeared in many previous relaying MAC protocols (e.g. [2], [4], [18]). The relay contention window is shown in Fig. 3. There are 5 sub-windows which are dedicated to nodes that meet listed requirements for data rates. Sub-window size is fixed with a constant value of SIFSs and the size represents number of slots dedicated to announcing relays' willingness for cooperation.

Once the source's timer expires the first, the source starts transmitting DATA frame, causing all other potential relays to back off. In case that, a relay's timer expires before source's timer, it broadcasts Relay Acknowledgment (RA) frame which is similar to CTS. After sensing any RA frame all potential relays quit from competition and wait until the channel is free again, the source estimates the maximum data rate which can be reached on link to the acknowledged relay. Since then, remaining nodes and the destination node expect relayed transmission. In case of collision in transmission of RAs, the source will detect the collision and start transmitting DATA

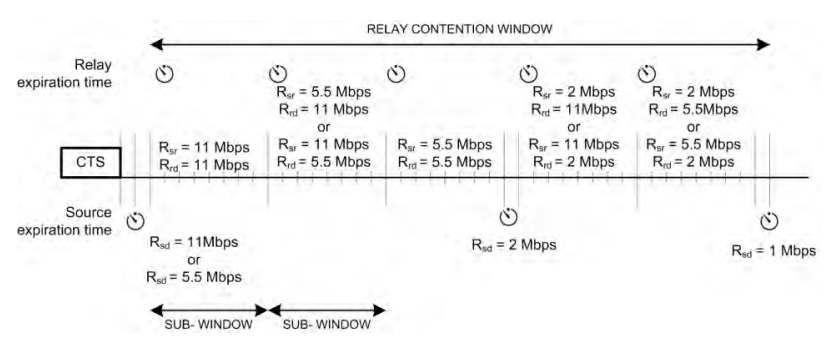

Figure 3. Contention window divided into 5 sub-windows of fixed sizes. 
frame at originally estimated data rate. Successful sending of DATA frame (directly or via relay) to destination node is confirmed with standard ACK message. To distinguish the relayed transmission, the source announces the relay address utilizing the 4th address field of MAC header.

In the 802.11 DCF protocol, any node overhearing either the RTS or the CTS extracts information from a frame and updates its network allocation vector (NAV), which is designed to indicate the time period reserved for end-to-end data transmission. Until the NAV expires, the node defers its own transmission. In order to implement relaying MAC protocol, its desired to design a new scheme of the NAV assignment. The goal is to ensure NAV updates at every node which receives either RTS, CTS, RA or data frame indicating it is duration in MAC header.

\section{ENERGY CONSUMPTION ANALYSIS}

In accordance to [8] and [9] power required for performing communication with Aironet PC4800 PCMCIA WLAN adapter can be approximated as $1900 \mathrm{~mW}$ for transmission mode and $1350 \mathrm{~mW}$ for receiving and idle ("waiting") mode. Although, these values can slightly vary in relation to device manufacturer and utilized data rates, without loss of generality, we consider the power consumption is fixed. The energy consumption $E$ is calculated as $E=P \cdot t$, where $P$ is the power of the instantaneous mode and $t$ the time of remaining in the instantaneous mode.

Taking into account $802.11 \mathrm{~b}$ system, transmission rate of control packets as $1 \mathrm{Mbps}$ and DSSS at physical level with long PLCP preamble $(192 \mu s)$ duration time, the energy consumption of control packet is presented in Table I.

Table I

ENERGY CONSUMPTION FOR RTS, CTS AND ACK FRAMES

\begin{tabular}{lcccc}
\hline Frame & Size & Duration & Tx energy & Rx energy \\
\hline RTS & $20 B$ & $160+192=352 \mu \mathrm{s}$ & $668.8 \mu \mathrm{J}$ & $475.2 \mu \mathrm{J}$ \\
CTS,ACK & $14 B$ & $112+192=304 \mu \mathrm{s}$ & $577.6 \mu \mathrm{J}$ & $410.4 \mu \mathrm{J}$ \\
\hline
\end{tabular}

By analogy, energy consumption for transmitting and receiving of data frame can be calculated as it is shown in Table II. Assuming that the outgoing frame size is limited to 1500 bytes, the total size of the MAC frame equals to 1536 bytes. Additional 36 bytes are added in encapsulation process, where the $802.11 \mathrm{~b}$ MAC header adds 28 bytes and further 8 bytes are added by the SNAP encapsulation header in order to identify the network layer protocol. Payload which is usable at higher layers (i.e. transport layer), referred to as goodput, is equal to 1460 bytes (1500 - 40 bytes required for IP and TCP headers). For the sake of simplicity, energy requirements

Table II

ENERGY REQUIRED FOR SERVING 1536 BYTES DATA FRAME

\begin{tabular}{lccc}
\hline Data rate & Duration (with PLCP) & Tx energy & Rx energy \\
\hline $1 \mathrm{Mbps}$ & $12480 \mu \mathrm{s}$ & $23712 \mu \mathrm{J}$ & $16848 \mu \mathrm{J}$ \\
$2 \mathrm{Mbps}$ & $6336 \mu \mathrm{s}$ & $12038 \mu \mathrm{J}$ & $8554 \mu \mathrm{J}$ \\
$5.5 \mathrm{Mbps}$ & $2427 \mu \mathrm{s}$ & $4612 \mu \mathrm{J}$ & $3277 \mu \mathrm{J}$ \\
$11 \mathrm{Mbps}$ & $1310 \mu \mathrm{s}$ & $2489 \mu \mathrm{J}$ & $1769 \mu \mathrm{J}$ \\
\hline
\end{tabular}

during interframe spaces correspond to energy consumption in receiving mode and are equal to $13.5 \mu \mathrm{J}(10 \mu \mathrm{s})$ and $67.5 \mu \mathrm{J}$ $(50 \mu s)$ for SIFS and DIFS, respectively.

In accordance to values presented in Table I and Table II total energy consumption required for single data frame transaction between two nodes in pure $802.11 \mathrm{~b}$, where control and data rates are equal to $1 \mathrm{Mbps}$, is shown in Table III.

Table III

ENERGY CONSUMPTION ANALYSIS - NON-RELAYING TRANSMISSION

\begin{tabular}{lccc}
\hline & Duration & Transmitter & Receiver \\
\hline RTS & $352 \mu \mathrm{s}$ & $668.8 \mu \mathrm{J}$ & $475.2 \mu \mathrm{J}$ \\
CTS & $304 \mu \mathrm{s}$ & $410.4 \mu \mathrm{J}$ & $577.6 \mu \mathrm{J}$ \\
DATA & $12480 \mu \mathrm{s}$ & $23712 \mu \mathrm{J}$ & $16848 \mu \mathrm{J}$ \\
ACK & $304 \mu \mathrm{s}$ & $410.4 \mu \mathrm{J}$ & $577.6 \mu \mathrm{J}$ \\
3x SIFS & $30 \mu \mathrm{s}$ & $40.5 \mu \mathrm{J}$ & $40.5 \mu \mathrm{J}$ \\
\hline sum & $13470 \mu \mathrm{s}$ & $25242 \mu \mathrm{J}$ & $18519 \mu \mathrm{J}$ \\
\hline \multicolumn{3}{c}{ total: $43761 \mu \mathrm{J}$}
\end{tabular}

In terms of goodput, the system efficiency is $0.87 \mathrm{Mb} / \mathrm{s}$ and the energy efficiency equals to $0.27 \mathrm{Mb} / \mathrm{J}$. If there exists another node in range of transmitter and receiver (that requires to transmit data), it consumes the energy while it listens to all ongoing transactions. Therefore, the system requires extra energy of $18.19 \mathrm{~mJ}$ which yields about $0.08 \mathrm{Mb} / \mathrm{J}$ efficiency degradation. Moreover, the efficiency decreases while frame collisions and data retransmissions occur.

Table IV shows energy consumed in a self-enforcing relaying system with 3 nodes. 1 Mbps data rate between transmitter and receiver and $11 \mathrm{Mbps}$ links relay-transmitter and relayreceiver are possible. The most optimistic case occurs when

Table IV

ENERGY CONSUMPTION ANALYSIS FOR MOST OPTIMISTIC SCENARIO

\begin{tabular}{|c|c|c|c|c|}
\hline & Duration & Transmitter & Receiver & Relay \\
\hline RTS & $352 \mu \mathrm{s}$ & $668.8 \mu J$ & $475.2 \mu \mathrm{J}$ & $475.2 \mu \mathrm{J}$ \\
\hline CTS & $304 \mu \mathrm{s}$ & $410.4 \mu \mathrm{J}$ & $577.6 \mu \mathrm{J}$ & $410.4 \mu \mathrm{J}$ \\
\hline RA & $304 \mu \mathrm{s}$ & $410.4 \mu \mathrm{J}$ & $410.4 \mu \mathrm{J}$ & $577.6 \mu \mathrm{J}$ \\
\hline DATA $_{s r}$ & $1320 \mu \mathrm{s}$ & $2508 \mu J$ & $1782 \mu J$ & $1782 \mu \mathrm{J}$ \\
\hline DATA $_{r d}$ & $1320 \mu \mathrm{s}$ & $1782 \mu \mathrm{J}$ & $1782 \mu \mathrm{J}$ & $2508 \mu \mathrm{J}$ \\
\hline ACK & $304 \mu s$ & $410.4 \mu J$ & $577.6 \mu \mathrm{J}$ & $410.4 \mu \mathrm{J}$ \\
\hline 6x SIFS & $60 \mu \mathrm{s}$ & $81 \mu J$ & $81 \mu J$ & $81 \mu J$ \\
\hline sum & $3964 \mu \mathrm{s}$ & $6271 \mu \mathrm{J}$ & $5685.8 \mu \mathrm{J}$ & $6244.6 \mu \mathrm{J}$ \\
\hline
\end{tabular}

RA message is sent in the first sub-window after 2 SIFS from CTS. In such a scenario, goodput efficiency reaches $2.95 \mathrm{Mb} / \mathrm{s}$ with average energy consumption at level of $0.64 \mathrm{Mb} / \mathrm{J}$. For case when RA is transmitted after 10 SIFS starting from CTS these values are $2.91 \mathrm{Mb} / \mathrm{s}$ and $0.63 \mathrm{Mb} / \mathrm{J}$ respectively. Finally, relative gain reaches almost $140 \%$ for energy and $240 \%$ for goodput efficiency.

\section{Simulation Results}

To examine the efficiency of the self-enforcing relaying strategy, Matlab-based event-driven simulator was implemented. All considered scenarios (S1-S4) comprise 4 node topology (shown in Fig. 4) with fixed SNRs yielding fixed data rates between them. Two basic transmission demands are defined separately between node pairs 1-2 and 3-4, by 
manipulating channel qualities between the nodes we obtain different data rates as in Table $\mathrm{V}$.

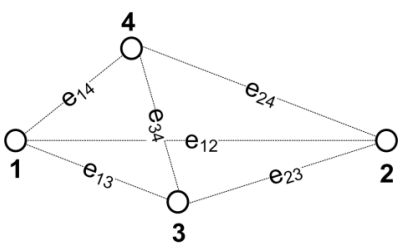

Figure 4. Network topology.

Assuming permanent transmission between nodes 1 and 2, results compare goodput efficiency (Mbps) and energy consumption as a function of payload bits per joule for realizing the demand on the link 3 and 4. Note that, $1 \mathrm{Mbps}$ and $11 \mathrm{Mbps}$ channels are always assumed in transmission between 1-2 $\left(e_{12}\right)$ and 3-4 $\left(e_{34}\right)$, respectively.

Figures 5-8 present evaluation for all scenarios and show influence of sub-window size on energy and goodput efficiencies obtained for realizing demand of nodes 3-4. Noncooperative benchmarks refer to the system based on $802.11 \mathrm{~b}$ with the following assumptions: (i) long PLCP preamble is used at physical layer, due to the fact that support of the long PLCP preamble at physical level is mandatory and is the default setting on most devices [16]; (ii) the exponential random backoff algorithm [13] is applied in order to provide collision avoidance; (iii) the rest of system's parametesr are listed in Table VI.

Link qualities in scenarios $S 1, S 2$ and $S 3$ motivate nodes 3 and 4 to help in relaying frames for transmission 1-2. These qualities are defined symmetrically in order to ensure some RA collisions are possible. In cases where sub-window size is 1 , two potential relays $(3,4)$ announce their willingness for cooperation by sending RA frame simultaneously and obvious collision of the RAs appears (a single slot is dedicated for broadcasting RA). These collisions fired for every RTS/CTS handshaking reduce transmission efficiency by increased duration time of total end-to-end transaction (from RTS to ACK) and consume more energy for delivering the same number of useful bytes in relation to 802.11 b. However, for $S 1-S 3$ some gain in energy consumption is observed starting from sub-window size equals to 2 where probability of collision of RAs is quite high and equals to $50 \%$. Thus, even if half of the frames are relayed, the energy savings are noticeable. Increased size of the sub-window in the system provides lower probability of collision while it may result in extended waiting time for proceeding transmission and eventually increased energy consumption.

Generally, the efficiency of self-enforcing relaying protocol is improved in comparison to basic $802.11 \mathrm{~b}$. This result is explicitly observed especially for instances with relays which can provide relatively high data rates on both links, fromsource and to-destination $(S 1, S 2)$. In case of $S 1$ (Fig. 5), the system efficiency of energy consumption can reach $350 \%$ of non-cooperative transmission. For scenario $S 2$ shown in
Table V

SYSTEM PARAMETERS APPLIED FOR SIMULATIONS

\begin{tabular}{l|c|cccc|c}
\hline Scenario & $\boldsymbol{e}_{\mathbf{1 2}}$ & $\boldsymbol{e}_{\mathbf{1 3}}$ & $\boldsymbol{e}_{\mathbf{1 4}}$ & $\boldsymbol{e}_{\mathbf{2 3}}$ & $\boldsymbol{e}_{\mathbf{2 4}}$ & $\boldsymbol{e}_{\mathbf{3 4}}$ \\
\hline S1 & $1 \mathrm{Mbps}$ & 11 & 11 & 11 & 11 & 11 \\
S2 & 1 & 5.5 & 5.5 & 5.5 & 5.5 & 11 \\
S3 & 1 & 2 & 5.5 & 5.5 & 2 & 11 \\
S4 & 1 & 1 & 1 & 1 & 1 & 11 \\
\hline
\end{tabular}

Table VI

SYSTEM PARAMETERS APPLIED FOR SIMULATIONS

\begin{tabular}{lc|lc}
\hline Parameter & Value & Parameter & Value \\
\hline PHY preamble & $192 \mu \mathrm{s}$ & DATA frame & $1536 \mathrm{~B}$ \\
RTS & $20 B, 352 \mu \mathrm{s}$ & DATA payload & $1460 \mathrm{~B}$ \\
CTS, ACK, RA & $14 B, 304 \mu \mathrm{s}$ & $\mathrm{CW}_{\min }$ & 4 \\
SIFS & $10 \mu \mathrm{s}$ & $\mathrm{CW}_{\max }$ & 10 \\
DIFS & $50 \mu \mathrm{s}$ & Sub Window Size & $1-20$ \\
Time Slot & $20 \mu \mathrm{s}$ & $T_{x}$ power & $1.9 \mathrm{~W}$ \\
Control rate & $1 \mathrm{Mbps}$ & $R_{x}$ power & $1.35 \mathrm{~W}$ \\
Data Rate & $1,2,5.5,11 \mathrm{Mbps}$ & Waiting for channel & $1.35 \mathrm{~W}$
\end{tabular}

Fig. 6, the energy efficiency is improved by about $160 \%$. Results obtained for $S 3$ in Fig. 7 indicate that, relays which are supposed to acknowledge their willingness to cooperation in the last 5th sub-window provide even two times better performance in relation to $802.11 \mathrm{~b}$.

The least promising situation is illustrated in Fig. 8 (scenario $S 4)$. The instantaneous source node of link 1-2 is unable to decide whether any relay is available and has to wait 5 subwindows for its timer expiration. During this time, energy is consumed as in receiving/idle mode and it generates extra overhead, as eventually, the transmission is performed in noncooperative scheme. In this case, energy-efficiency degradation is evaluated to averaged $9-10 \%$.

\section{CONCLUSION}

In this paper we proposed and examined simple selfenforcing relaying protocol based on $802.11 \mathrm{~b}$. The main idea behind the self-enforcement is to trigger decisions about relaying in distributed way without any external agents. This selfenforcement incorporates internal motivation of users. Simulation results indicate that, all scenarios in which efficiency can be improved, eventually, increase energy gain from almost 0.6 $M b / J$ (which constitutes for $250 \%$ gain over non-cooperative scenario) to $0.23 \mathrm{Mb} / \mathrm{J}$ for the scenario with weaker channel qualities between source to relay and relay to destination. The worst case scenario, where selection of relay fails, brings an absolute cost in the range of $0.01-0.03 \mathrm{Mb} / \mathrm{J}$, which is still low enough in comparison with the possible energy saving gain. The results clearly show that, in general, selfenforcing relaying outperforms simple 802.11 b mechanisms in terms of energy efficiency, especially for relays available with relatively high channel quality. As far as transmit mode has relatively marginal impact on overall energy consumption, it might be worth participating in relaying of concurrent frames and spending additional energy on being "mid-transmitter" instead of "wasting" energy in receiving/idle state with regard to gaining faster access to the channel. 


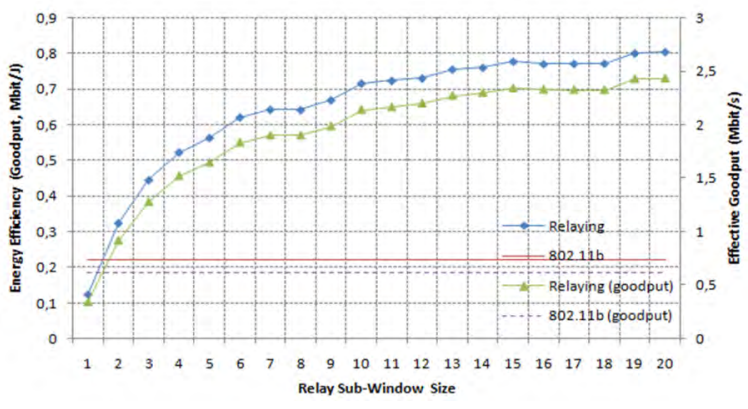

Figure 5. Results for scenario $S 1$.

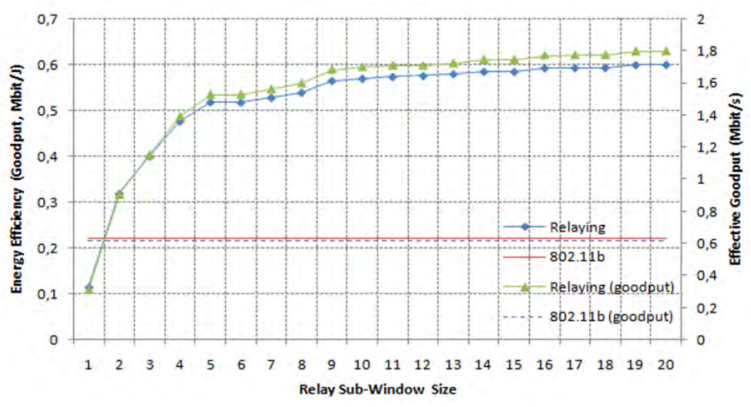

Figure 6. Results for scenario $S 2$.

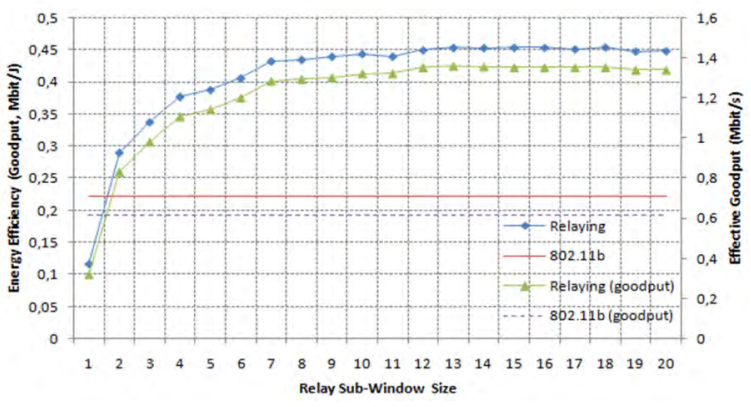

Figure 7. Results for scenario $S 3$.

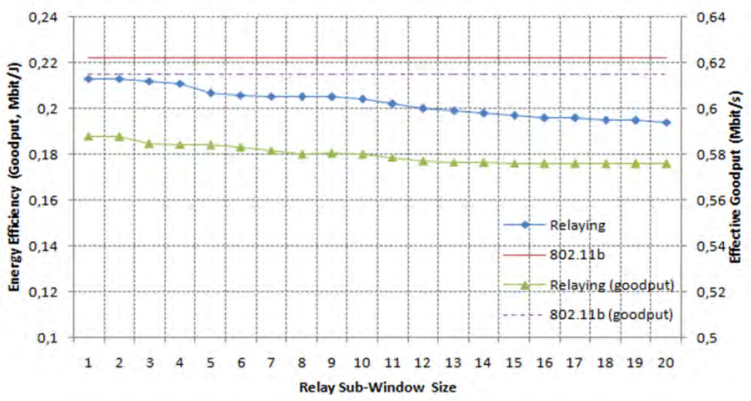

Figure 8. Results for scenario $S 4$.

\section{ACKNOWLEDGMENT}

The research leading to these results has received funding from the European Community's Seventh Framework Programme [FP7/2007-2013] under grant agreement $\mathrm{N}^{\circ} 248577$ [C2POWER] and grant agreement $\mathrm{N}^{\circ} 257626$ [ACROPOLIS].

\section{REFERENCES}

[1] H. Adam, C. Bettstetter, and S.-M. Senouci, "Adaptive Relay Selection in Cooperative Wireless Networks," in PIMRC. IEEE, 2008, pp. 1-5.

[2] A. Azgin, Y. Altunbasak, and G. AlRegib, "Cooperative MAC and Routing Protocols for Wireless Ad Hoc Networks," in IEEE GLOBECOM Conference, vol. 5, St. Louis, 2005, pp. 2854-2859.

[3] M. M. Carvalho, C. B. Margi, K. Obraczka, and J. J. Garcia-LunaAceves, "Modeling Energy Consumption in Single-Hop IEEE 802.11 Ad Hoc Networks," in Proc. 13th Int. Conf. Computer Communications and Networks ICCCN 2004, 2004, pp. 367-372.

[4] C. Cetinkaya and F. Orsun, "Cooperative Medium Access Protocol for Dense Wireless Networks," in The Third Annual Mediterranean Ad Hoc Networking Workshop - Med Hoc Net 2004, Bordum, Turkey, 2004.

[5] Y. Chen, G. Yu, P. Qiu, and Z. Zhang, "Power-Aware Cooperative Relay Selection Strategies in Wireless Ad Hoc Networks," in PIMRC, 2006, pp. $1-5$.

[6] C.-T. Chou, J. Yang, and D. Wang, "Cooperative MAC Protocol with Automatic Relay Selection in Distributed Wireless Networks," in PERCOMW '07: Proceedings of the Fifth IEEE International Conference on Pervasive Computing and Communications Workshops. Washington, DC, USA: IEEE Computer Society, 2007, pp. 526-531.

[7] S. Cui, A. J. Goldsmith, and A. Bahai, "Energy-Efficiency of MIMO and Cooperative MIMO Techniques in Sensor Networks," vol. 22, no. 6, pp. 1089-1098, 2004.

[8] J.-P. Ebert, S. Aier, G. Kofahl, E. Becker, B. Burns, and A. Wolisz, "TKN Technical Reports Series, Technical University Berlin," 2002.

[9] L. M. Feeney and M. Nilsson, "Investigating the Energy Consumption of a Wireless Network Interface in an Ad Hoc Networking Environment," in In IEEE Infocom, 2001, pp. 1548-1557.

[10] T. Guo and R. A. Carrasco, "CRBAR: Cooperative Relay-Based Auto Rate MAC for Multirate Wireless Networks," IEEE Transactions on Wireless Communications, vol. 8, no. 12, pp. 5938-5947, 2009.

[11] M. Heusse, F. Rousseau, G. Berger-Sabbatel, and A. Duda, "Performance Anomaly of 802.11b," in Proc. INFOCOM 2003. Twenty-Second Annual Joint Conf. of the IEEE Computer and Communications. IEEE Societies, vol. 2, 2003, pp. 836-843.

[12] T. E. Hunter and A. Nosratinia, "Cooperation Diversity Through Coding," in Proc. IEEE Int Information Theory Symp, 2002.

[13] "Wireless LAN Medium Access Control (MAC) and Physical Layer (PHY) Specifications," IEEE Standard 802.11, June 1999.

[14] J. N. Laneman, D. N. C. Tse, and G. W. Wornell, "Cooperative Diversity in Wireless Networks: Efficient Protocols and Outage Behavior," vol. 50, no. 12 , pp. $3062-3080,2004$.

[15] J. N. Laneman and G. W. Wornell, "Distributed Space-Time-Coded Protocols for Exploiting Cooperative Diversity in Wireless Networks," vol. 49, no. 10, pp. 2415-2425, 2003.

[16] B. G. Lee and S. Choi, Broadband Wireless Access and Local Networks: Mobile WiMax and WiFi. Artech House, 2008.

[17] R. Madan, N. B. Mehta, A. F. Molisch, and J. Zhang, "Energy-Efficient Cooperative Relaying over Fading Channels with Simple Relay Selection," in Proc. IEEE Global Telecommunications Conf. GLOBECOM '06, 2006, pp. 1-6.

[18] M. Offner and H. Adam, "Cooperative Media Access Control in Wireless Networks," in Proceedings of the Junior Scientist Conference 2008, Vienna, 2008, pp. 47-48.

[19] T. Rappaport, Wireless Communications: Principles and Practice, 2nd ed. Upper Saddle River, NJ, USA: Prentice Hall PTR, 2001.

[20] A. Sendonaris, E. Erkip, and B. Aazhang, "User Cooperation Diversity. Part I. System Description," vol. 51, no. 11, pp. 1927-1938, 2003.

[21] A. Stefanov and E. Erkip, "Cooperative Coding for Wireless Networks," vol. 52, no. 9, pp. 1470-1476, 2004.

[22] _ _ "Cooperative Space-Time Coding for Wireless Networks," vol. 53, no. 11 , pp. 1804-1809, 2005.

[23] G. Zheng, K.-K. Wong, A. Paulraj, and B. Ottersten, "CollaborativeRelay Beamforming With Perfect CSI: Optimum and Distributed Implementation," vol. 16, no. 4, pp. 257-260, 2009.

[24] Z. Zhou, S. Zhou, J.-H. Cui, and S. Cui, "Energy-Efficient Cooperative Communication based on Power Control and Selective Relay in Wireless Sensor Networks," in Proc. IEEE Military Communications Conf. MILCOM 2007, 2007, pp. 1-7. 